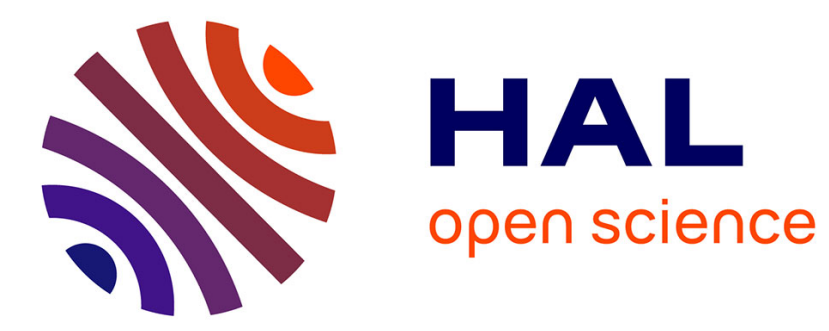

\title{
Ségrégation du carbone et du silicium sur la face (100) d'un alliage Fe-Si interprétation par le modèle de Guttmann
}

\author{
M. Essouni, A. Mosser
}

\section{To cite this version:}

M. Essouni, A. Mosser. Ségrégation du carbone et du silicium sur la face (100) d'un alliage Fe-Si interprétation par le modèle de Guttmann. Revue de Physique Appliquée, 1986, 21 (4), pp.257-261. 10.1051/rphysap:01986002104025700 . jpa-00245438

\section{HAL Id: jpa-00245438 https://hal.science/jpa-00245438}

Submitted on 1 Jan 1986

HAL is a multi-disciplinary open access archive for the deposit and dissemination of scientific research documents, whether they are published or not. The documents may come from teaching and research institutions in France or abroad, or from public or private research centers.
L'archive ouverte pluridisciplinaire HAL, est destinée au dépôt et à la diffusion de documents scientifiques de niveau recherche, publiés ou non, émanant des établissements d'enseignement et de recherche français ou étrangers, des laboratoires publics ou privés. 


\title{
REVUE DE PHYSIQUE APPLIQUÉE
}

Revue Phys. Appl. 21 (1986) 257-261

AVRIL 1986, PAGE 257

Classification

Physics Abstracts

$64.75-81.30 \mathrm{M}$

\section{Ségrégation du carbone et du silicium sur la face (100) d'un alliage Fe-Si interprétation par le modèle de Guttmann}

\author{
M. Essouni et A. Mosser \\ Université Louis Pasteùr, Laboratoire de Cristallographie, Equipe d'Etude des Surfaces, U.A. 795 du C.N.R.S. \\ 4, rue Blaise Pascal, 67000 Strasbourg, France
}

(Reçu le 9 décembre 1985, accepté le 9 janvier 1985)

\begin{abstract}
Résumé. - Les résultats expérimentaux concernant la ségrégation du carbone et du silicium à la surface (100) d'un alliage monocristallin de $\mathrm{Fe}-6$ at \% Si ont été interprétés par un modèle de type Guttmann. Ce modèle relatif aux solutions ternaires de substitution décrit les phénomènes de ségrégation en termes de compétition avec répulsion entre le carbone et le silicium. Un bon accord entre les courbes expérimentales obtenues par de Rugy par AES et les courbes calculées à l'aide du modèle de Guttmann a été obtenu. La valeur du coefficient d'interaction en surface entre le carbone et le silicium a été trouvée égale à $-23000 \mathrm{~J} / \mathrm{mole}$. De plus, l'enthalpie de ségrégation du carbone dans le fer déterminée : $92000 \mathrm{~J} / \mathrm{mole}$, est en bon accord avec celle trouvée par Grabke sur le fer pur.
\end{abstract}

\begin{abstract}
Carbon and silicon experimental segregation results on the (100) face of $\mathrm{Fe}-6 \mathrm{at} \% \mathrm{Si}$ alloy were interpreted with a Guttmann segregation model. This model which concerns ternary substitutional solutions, describes segregation phenomena in terms of sites competition and repulsive interaction between carbon and silicon atoms. Good agreement was obtained between experimental curves obtained by de Rugy with AES and curves calculated with Guttmann's model. The surface repulsive interaction coefficient between carbon and silicon was found equal to $-23000 \mathrm{~J} / \mathrm{mole}$. In addition the segregation enthalpy of carbon in iron obtained at the same time was equal to $92000 \mathrm{~J} / \mathrm{mole}$ which is in good agreement with the value found by Grabke in pure iron.
\end{abstract}

\section{Introduction.}

La ségrégation des impuretés, dans les métaux et alliages métalliques, est un phénomène qui joue souvent un rôle décisif dans des situations aussi différentes que la catalyse hétérogène [1], la corrosion [2-4] ou bien la fragilisation de trempe des métaux et alliages d'ingénierie [5-10]. Aussi, l'étude de ces phénomènes de ségrégation a-t-elle pris un essor particulier surtout depuis le développement des techniques d'analyse des surfaces et notamment la spectroscopie d'électrons Auger. De même le travail théorique accompli dans ce domaine a été abondant et plusieurs modèles ont été proposés dans le but de décrire, prévoir et quantifier ces phénomènes aussi bien en termes d'équilibre que de cinétique de ségrégation [11-17].

Mais si les systèmes binaires ont reçu une attention particulière de par les nombreux travaux qui leur ont été consacré, il n'en va pas de même pour les systèmes à plusieurs constituants et notamment les systèmes ternaires. Le modèle développé par Guttmann [18] constitue cependant une approche originale de la ségrégation dans ces systèmes en rendant compte, en particulier, de l'influence des interactions chimiques qui peuvent exister d'une part entre solutés et d'autre part entre chaque soluté et le substrat.

A l'aide du formalisme de la thermodynamique classique et en utilisant l'approche quasichimique des solutions régulières ainsi que les équations de Darken [19] pour l'expression de l'enthalpie de mélange du système, Guttmann propose les isothermes d'équilibre suivants dans le cas de compétition sur les sites de surface entre atomes de solutés 1 et 2 dans le substrat 3

$$
\frac{X_{1}^{\mathrm{s}}}{1-X_{1}^{\mathrm{s}}-X_{2}^{\mathrm{s}}}=\frac{X_{1}^{\mathrm{v}}}{1-X_{1}^{\mathrm{v}}-X_{2}^{\mathrm{v}}} \exp \left(\frac{\Delta G_{1}}{R T}\right)
$$

de même pour le soluté 2

$$
\frac{X_{2}^{\mathrm{s}}}{1-X_{1}^{\mathrm{s}}-X_{2}^{\mathrm{s}}}=\frac{X_{2}^{\mathrm{v}}}{1-X_{2}^{\mathrm{v}}-X_{1}^{\mathrm{v}}} \exp \left(\frac{\Delta G_{2}}{R T}\right)
$$

$X_{1(2)}^{\mathrm{s}}$ : concentration de l'élément 1 (2) en surface

$X_{1(2)}^{\mathrm{v}}$ : concentration volumique de l'élément 1 (2) $\Delta G_{1(2)}$ : enthalpie libre de ségrégation de 1 (2) 
avec

$\Delta G_{1}=\Delta G_{1}^{0}+2 \alpha_{13}\left(X_{1}^{\mathrm{v}}-X_{1}^{\mathrm{s}}\right)+\alpha^{\prime}\left(X_{2}^{\mathrm{s}}-X_{2}^{\mathrm{v}}\right)$

$\Delta G_{2}=\Delta G_{2}^{0}+2 \alpha_{23}\left(X_{2}^{\mathbf{v}}-X_{2}^{\mathrm{s}}\right)+\alpha^{\prime}\left(X_{1}^{\mathrm{s}}-X_{1}^{\mathrm{v}}\right)$

et

$$
\alpha^{\prime}=\alpha_{12}-\alpha_{23}-\alpha_{13}
$$

Les termes $\alpha_{i j}$ sont les coefficients d'interactions binaires entre proches voisins définis de la manière suivante :

$$
\alpha_{i j}=N_{0} Z\left[e_{i j}-\frac{1}{2}\left(e_{j j}+e_{i i}\right)\right]
$$

où $N_{0}$ est le nombre d'Avogadro, $Z$ le nombre de coordination et $e_{i j}$ l'énergie de liaison entre $i$ et $j$. Pour simplifier, on considère que : $\alpha_{i j}^{\mathbf{s}}=\alpha_{i j}^{\mathrm{v}}$ (où $\mathrm{s}$ désigne la surface et $v$ le volume).

Dans les équations (3) et (4), le terme $\Delta G_{1}^{0}$ est appelé terme de McLean et concerne la ségrégation binaire du soluté (1) dans le solvant (3). Il ne tient compte que de la variation de l'énergie libre du système d'origine non configurationnelle (énergie élastique, vibrationnelle) quand un atome du soluté remplace un atome du solvant en surface [18].

Le deuxième terme appelé par Guttmann « terme de Fowler " permet de rendre compte de la contribution à l'énergie de ségrégation des interactions chimiques entre le soluté 1 (2) et le solvant 3 si elles existent; l'influence de ce terme reste toutefois de peu d'importance dans le cas où il existe de fortes interactions entre les solutés 1 et 2 .

Le troisième terme de l'équation (3) [4] contient le coefficient d'interaction binaire $\alpha^{\prime}=\alpha_{12}-\alpha_{23}-\alpha_{13}$ qui traduit l'excès des interactions entre les solutés 1 et 2 par rapport à celles qui existent entre le solvant et chacun des deux solutés. Ainsi si $\alpha^{\prime}>0$ c'est-à-dire lorsque la formation des liaisons 1-2 est préférentielle; les équations (2) et (4) montrent que la ségrégation de l'un des deux solutés, en l'occurrence le soluté ayant la plus forte énergie de ségrégation $\Delta G^{0}$, entraîne celle du deuxième soluté, créant ainsi un effet de synergie. Il est significatif pour ces systèmes que même si l'un des deux constituants ne possède pas d'énergie de ségrégation propre lorsqu'il est en solution binaire dans le solvant, il est entraîné vers la surface du fait de l'attraction exercée par l'autre élément. Parmi les nombreux exemples qui illustrent ce cas, on peut citer les systèmes $\mathrm{Fe}-\mathrm{Mo}-\mathrm{C}$ et $\mathrm{Fe}-\mathrm{Cr}-\mathrm{N}$ où, à la fois, le molybdène et le chrome ne ségrègent pas dans le fer, mais qui, en présence de carbone pour l'un et d'azote pour l'autre ségrègent fortement en surface [20].

Le cas particulier de $\alpha^{\prime}<0$, c'est-à-dire lorsqu'il y a répulsion entre les deux solutés, nous intéresse au premier chef. En effet, le travail que nous avons effectué concerne le système $\mathrm{Fe}-\mathrm{Si}-\mathrm{C}$ qui est donné comme exemple type pour illustrer ce dernier cas du fait de la forte répulsion qui existe entre le silicium et le carbone dans une matrice de fer comme l'ont montré les expériences de Darken [21]. D'après cet auteur, lorsque l'on maintient un couple de diffusion $\mathrm{Fe}-\mathrm{C} / \mathrm{Fe}-\mathrm{C}-3$, $80 \%$ Si de même teneur en carbone à $1050^{\circ} \mathrm{C}$, il se produit un phénomène de diffusion inversée, où le carbone migre vers la région pauvre en silicium; ce qui montre bien, qu'en solution solide dans le fer, le silicium a pour effet d'augmenter l'activité et donc le potentiel chimique du carbone.

Un comportement analogue de ces deux éléments à la surface du fer a été décrit récemment par de Rugy [22]. Les résultats expérimentaux, qui ont été réalisé par AES sur la face (100) d'un échantillon monocristallin de $\mathrm{Fe}-6$ at $\% \mathrm{Si}$, montrent que le carbone ségrège fortement à $500^{\circ} \mathrm{C}$ pour saturer la surface. Lorsque la température augmente, le carbone se dissout dans le volume et est remplacé en surface par le silicium. Il s'avère par ailleurs, d'après les mêmes résultats que, plus la concentration volumique en carbone est importante, plus la température de dissolution de celui-ci est grande. De plus, sur un échantillon exempt de carbone et autres impuretés, l'auteur montre que le silicium ségrège et forme en surface une couche de ségrégation qui, à saturation, possède une structure ordonnée $\mathrm{C}(2 \times 2)$ mise en évidence par Leed. Dans les mêmes conditions, l'enthalpie libre de ségrégation du silicium a été déterminée à l'aide du modèle de Langmuir-McLean et trouvée égale à :

$$
\Delta G_{\mathrm{Si}}=48000-15 T \mathrm{~J} / \mathrm{mole}
$$

\section{Application du modèle de Guttmann au système Fe-Si-C.}

Le but de ce travail est de montrer que les résultats expérimentaux obtenus par de Rugy sur le système Fe-Si-C peuvent être interprétés par le modèle de Guttmann relatif à la ségrégation dans les solutions ternaires de substitution.

Si l'on applique les équations (1), (2), (3) et (4) au système $\mathrm{Fe}-\mathrm{Si}-\mathrm{C}$ on trouve :

$$
\frac{X_{\mathrm{Si}}}{1-X_{\mathrm{Si}}-X_{\mathrm{C}}}=\frac{C_{\mathrm{Si}}}{1-C_{\mathrm{Si}}-C_{\mathrm{c}}} \exp \left(\Delta G_{\mathrm{Si}} / R T\right)
$$

de même pour le carbone

$$
\frac{X_{\mathrm{C}}}{1-X_{\mathrm{Si}}-X_{\mathrm{C}}}=\frac{C_{\mathrm{C}}}{1-C_{\mathrm{Si}}-C_{\mathrm{C}}} \exp \left(\Delta G_{\mathrm{C}} / R T\right)
$$

où

$X_{\mathrm{Si}(\mathrm{C})}=\frac{C_{\mathrm{Si}(\mathrm{C})}^{\mathrm{S}}}{\left(C_{\mathrm{Si}(\mathrm{C}))_{\mathrm{sat}}}^{\mathrm{S}}\right.}$ est le taux de couverture à l'équilibre;

$C_{\mathrm{Si}}^{\mathbf{S}}$ et $\left(C_{\mathrm{Si}}^{\mathbf{S}}\right)_{\text {sat }}$ sont respectivement la concentration superficielle à l'équilibre et la concentration superficielle à saturation $C_{\mathrm{Si}(\mathrm{C})}$ est la concentration volumique en $\mathrm{Si}(\mathrm{C})$

$\Delta G_{\mathrm{Si}(\mathrm{C})}$ est l'enthalpie libre de ségrégation du $\mathrm{Si}(\mathrm{C})$ 
avec

$$
\Delta G_{\mathrm{Si}}=\Delta G_{\mathrm{Si}}^{0}+2 \alpha_{\mathrm{Fe}-\mathrm{Si}}\left(C_{\mathrm{Si}}-X_{\mathrm{Si}}\right)+\alpha^{\prime}\left(X_{\mathrm{C}}-C_{\mathrm{C}}\right)
$$

de même pour le carbone

$$
\Delta G_{\mathrm{C}}=\Delta G_{\mathrm{C}}^{0}+2 \alpha_{\mathrm{Fe}-\mathrm{C}}\left(C_{\mathrm{C}}-X_{\mathrm{C}}\right)+\alpha^{\prime}\left(X_{\mathrm{Si}}-C_{\mathrm{Si}}\right)
$$

où :

$$
\alpha^{\prime}=\alpha_{\mathrm{Si}-\mathrm{C}}-\alpha_{\mathrm{Fe}-\mathrm{Si}}-\alpha_{\mathrm{Fe}-\mathrm{C}}
$$

$\Delta G_{\mathrm{Si}}^{0}$ et $\Delta G_{\mathrm{C}}^{0}$ sont respectivement l'enthalpie libre de ségrégation du silicium et du carbone dans le fer pur. Elles ont été déterminées d'une part par Grabke et al. [23] pour le carbone sur la face (100) du fer $\left(\Delta G_{\mathrm{C}}^{0} \simeq 84,8 \mathrm{~kJ} /\right.$ mole et d'autre part par de Rugy [22] pour le silicium sur la face (100) d'un alliage Fe-6 at \% $\mathrm{Si}\left(\Delta G_{\mathrm{Si}}^{0}=48000-15 T \mathrm{~J} / \mathrm{mole}\right)$.

Ces deux valeurs ont été déterminées à l'aide de l'isotherme de Langmuir McLean et ceci nous amène légitimement à négliger le " terme de Fowler " dans les équations de Guttmann.

Les termes $\alpha^{\prime} C_{\mathrm{C}}$ et $\alpha^{\prime} C_{\mathrm{Si}}$ correspondent aux interactions entre le silicium et le carbone dans le volume. De ces deux termes, seul le second sera non négligeable $\mathrm{du}$ fait de la concentration volumique en silicium relativement importante. Finalement, nous pouvons écrire les équations de Guttmann pour le carbone et le silicium sous la forme simplifiée suivante :

$$
\begin{aligned}
& \Delta G_{\mathrm{C}}=\Delta G_{\mathrm{C}}^{0 \prime}+\alpha^{\prime} X_{\mathrm{Si}} \\
& \Delta G_{\mathrm{Si}}=\Delta G_{\mathrm{Si}}^{0}+\alpha^{\prime} X_{\mathrm{C}}
\end{aligned}
$$

avec

$$
\Delta G_{\mathbf{C}}^{0 \prime}=\Delta G_{\mathbf{C}}^{0}+\alpha^{\prime} C_{\mathrm{Si}} .
$$

Le calcul de $\alpha^{\prime}$ nécessite la connaissance des coefficients d'interaction $\alpha_{i j}$ pour les différents systèmes impliqués : $\mathrm{Fe}-\mathrm{C}, \mathrm{Fe}-\mathrm{Si}$ et $\mathrm{C}-\mathrm{Si}$. Ces coefficients peuvent être calculés, dans le cadre de l'approximation quasichimique des solutions régulières, si on connaît les enthalpies de mélange respectives puisque $\Delta H_{\mathrm{m}}^{i j}=$ $\alpha_{i j} X_{i} X_{j}$. Cependant, il est possible, comme le proposait Guttmann [18], d'évaluer les différents $\alpha_{i j}$ à partir des enthalpies standards de formation des composés stœechiométriques $i j$ correspondants, à l'aide de la formule :

$$
\alpha_{i j}=\frac{\Delta H_{i j}}{N_{i} N_{j}} .
$$

où $N_{i}$ et $N_{j}$ sont les fractions molaires de $i$ et de $j$.

Les valeurs des enthalpies standards de formation $\left(-\Delta H_{i j}\right)$ des composés $\mathrm{Fe}_{3} \mathrm{C}, \mathrm{Fe}-\mathrm{Si}$ et $\mathrm{Si}-\mathrm{C}$ trouvées dans la littérature sont respectivement : $-22,6 \mathrm{~kJ} /$ mole pour $\mathrm{Fe}_{3} \mathrm{C}, 80,2 \mathrm{~kJ} /$ mole pour $\mathrm{Fe}-\mathrm{Si}$ et $62,7 \mathrm{~kJ} /$ mole pour Si-C [24]. Les $\alpha_{i j}$ calculés à partir de ces valeurs sont alors :

$$
\alpha_{\mathrm{Fe}-\mathrm{C}}=-120,4 \mathrm{~kJ} / \mathrm{mole}, \quad \alpha_{\mathrm{Fe}-\mathrm{Si}}=321 \mathrm{~kJ} / \mathrm{mole}
$$

et

$$
\alpha_{\mathrm{Si}-\mathrm{C}}=250,8 \mathrm{~kJ} / \mathrm{mole},
$$

ce qui donne

$$
\alpha^{\prime}=+51 \mathrm{~kJ} / \text { mole }>0 \text {. }
$$

On constate que, le calcul fournit une valeur positive relativement importante pour $\alpha^{\prime}$ qui suggère une interaction attractive entre le silicium et le carbone, ce qui est en contradiction flagrante avec d'une part, les résultats expérimentaux de de Rugy et d'autre part, avec les travaux de Darken [21]. A notre avis, l'erreur provient de l'évaluation du coefficient $\alpha_{C-S i}$ auquel on ne peut accéder que par des mesures expérimentales dans le système ternaire $\mathrm{Fe}-\mathrm{Si}-\mathrm{C}$ [25].

A défaut de pouvoir calculer le terme $\alpha^{\prime}$, il est possible de déterminer sa valeur à partir des mesures expérimentales des taux de couvertures du silicium et du carbone à la surface d'échantillons Fe-Si contenant des concentrations différentes en carbone. En traçant $\operatorname{Ln} \frac{X_{\mathrm{Si}}}{1-X_{\mathrm{Si}}-X_{\mathrm{C}}}$ en fonction de $X_{\mathrm{C}}$ pour une température donnée, on obtient une droite dont la pente donne le facteur $\alpha^{\prime}$ correspondant à la répulsion en surface entre le silicium et le carbone. C'est ce que nous avons fait à partir des résultats expérimentaux de de Rugy. Cet auteur a réalisé en effet ses mesures par AES sur un échantillon monocristallin de Fe-6 at \% Si de face (100) contenant $40 \mathrm{ppm}$ de carbone en poids et ayant ensuite subit un traitement de décarburation amenant la composition en carbone à $C_{2}<40 \mathrm{ppm}$ puis à $C_{3}<C_{2}$. Malheureusement $C_{2}$ et $C_{3}$ n'ont pu être déterminées par cet auteur.

Nous avons reproduit sur la figure la les courbes expérimentales montrant l'évolution des taux de couverture du carbone et du silicium en fonction de la température pour chaque concentration en carbone. Le calcul de $\alpha^{\prime}$ qui en découle fournit des valeurs qui s'insèrent dans un intervalle de 31350 à $-23000 \mathrm{~J} /$ mole.

\section{Comparaison des courbes théoriques et expérimen- tales.}

En utilisant les équations (7), (8), (12) et (13), nous avons calculé, à l'aide d'une méthode d'itérations successives, les taux de couverture respectifs du carbone et du silicium pour des températures comprises entre $500^{\circ} \mathrm{C}$ et $1000^{\circ} \mathrm{C}$. Différentes valeurs de $\alpha^{\prime}$ et de $\Delta G_{\mathrm{C}}^{0 \prime}$ ont été testées pour $\alpha^{\prime}$ variant entre -31350 et $-16700 \mathrm{~J} / \mathrm{mole}$ et $\Delta G_{\mathrm{C}}^{0 \prime}$ entre 79400 et $100300 \mathrm{~J} /$ mole. Nous avons pris l'enthalpie de ségrégation du silicium dans le fer égale à $\Delta G_{\mathrm{Si}}^{0}=48000-15 T \mathrm{~J} /$ mole la concentration volumique du carbone égale à 60 ppm en fraction de site (correspondant à 40 ppm 
en poids, en supposant que le carbone occupe les sites octaédriques de la matrice ferritique) et enfin la concentration volumique du silicium égale à $6 \%$ at.

Dans ces conditions, le meilleur accord entre la courbe calculée et la courbe expérimentale est obtenu pour $\alpha^{\prime}=-23000 \mathrm{~J} / \mathrm{mole}$ et $\Delta G_{\mathrm{C}}^{0 \prime}=92000 \mathrm{~J} / \mathrm{mole}$ (en supposant nulle l'entropie de ségrégation du carbone).

Il faut remarquer que la valeur de $\Delta G_{\mathrm{C}}^{0 \prime}$ est comparable, aux erreurs expérimentales près, à la valeur de l'enthalpie de ségrégation obtenue par Grabke et al. [23] pour le carbone, sur la face (100) d'un échantillon monocristallin de fer $\left(\Delta G_{\mathrm{C}}^{0}=84850 \mathrm{~J} / \mathrm{mole}\right)$. Le

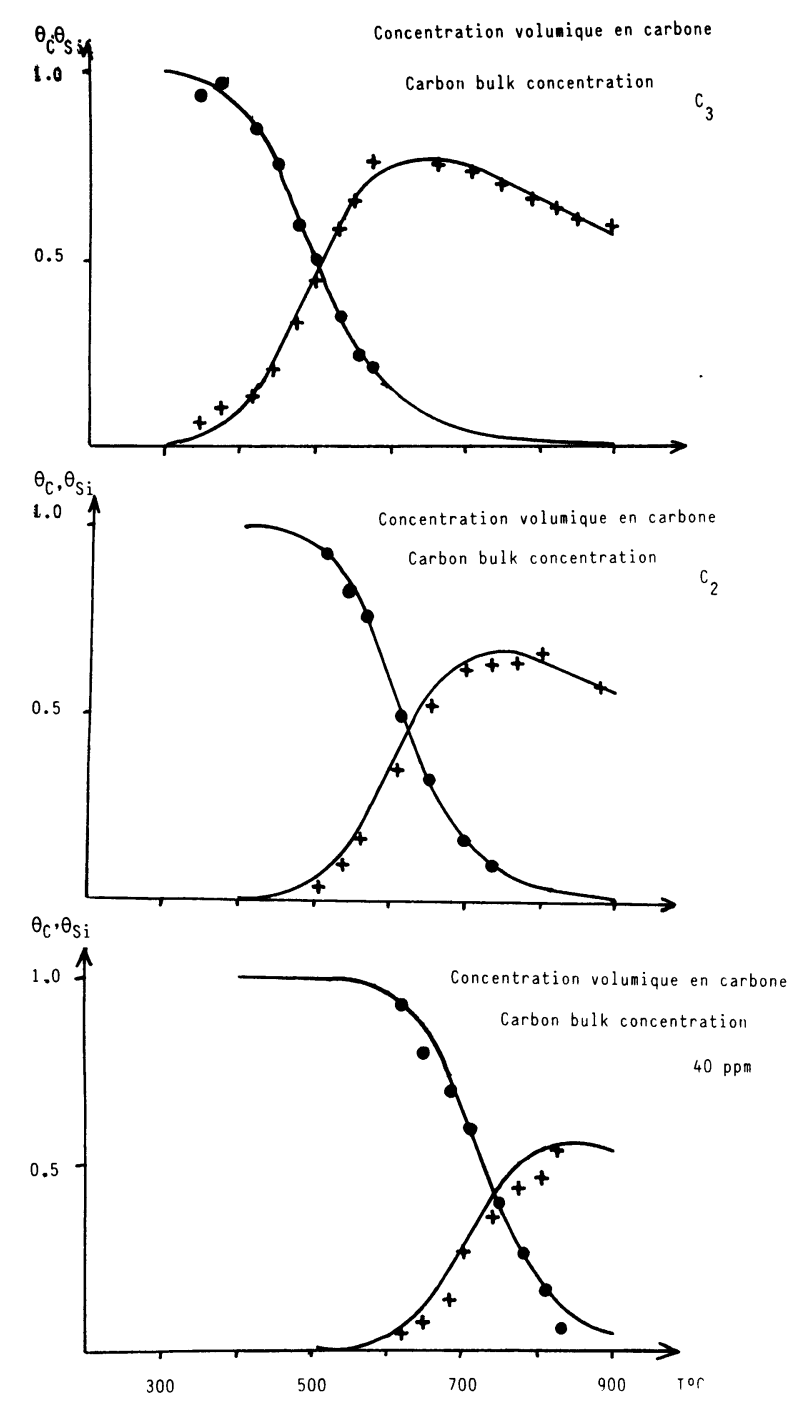

a)

Fig. 1a. - Courbes expérimentales donnant la variation du taux de couverture du carbone $\theta_{\mathrm{C}}(\cdot)$ et du silicium $\theta_{\mathrm{Si}}(+)$ en fonction de la température pour trois concentrations volumiques différentes en carbone d'après de Rugy [22].

b) Courbes calculées à l'aide du modèle de Guttmann donnant la variation du taux de couverture du carbone $\theta_{\mathrm{c}}($. et du silicium $\theta_{\mathrm{Si}}(+)$ pour des concentrations en carbone de 10,25 et $40 \mathrm{ppm}$. Les courbes en pointillés correspondent à $\alpha^{\prime}=0$. terme $\alpha^{\prime} C_{\mathrm{Si}}$ qui traduit la différence d'enthalpie de ségrégation du carbone dans l'alliage $\mathrm{Fe}-\mathrm{Si}$ et dans le fer pur est égale à $1670 \mathrm{~J} / \mathrm{mole}$ et améliore l'accord entre les deux valeurs précédentes.

Sur la figure 1, nous avons représenté d'une part les courbes expérimentales obtenues par de Rugy et d'autre part, les courbes calculées à l'aide du modèle de Guttmann relatif aux solutions ternaires de substi-
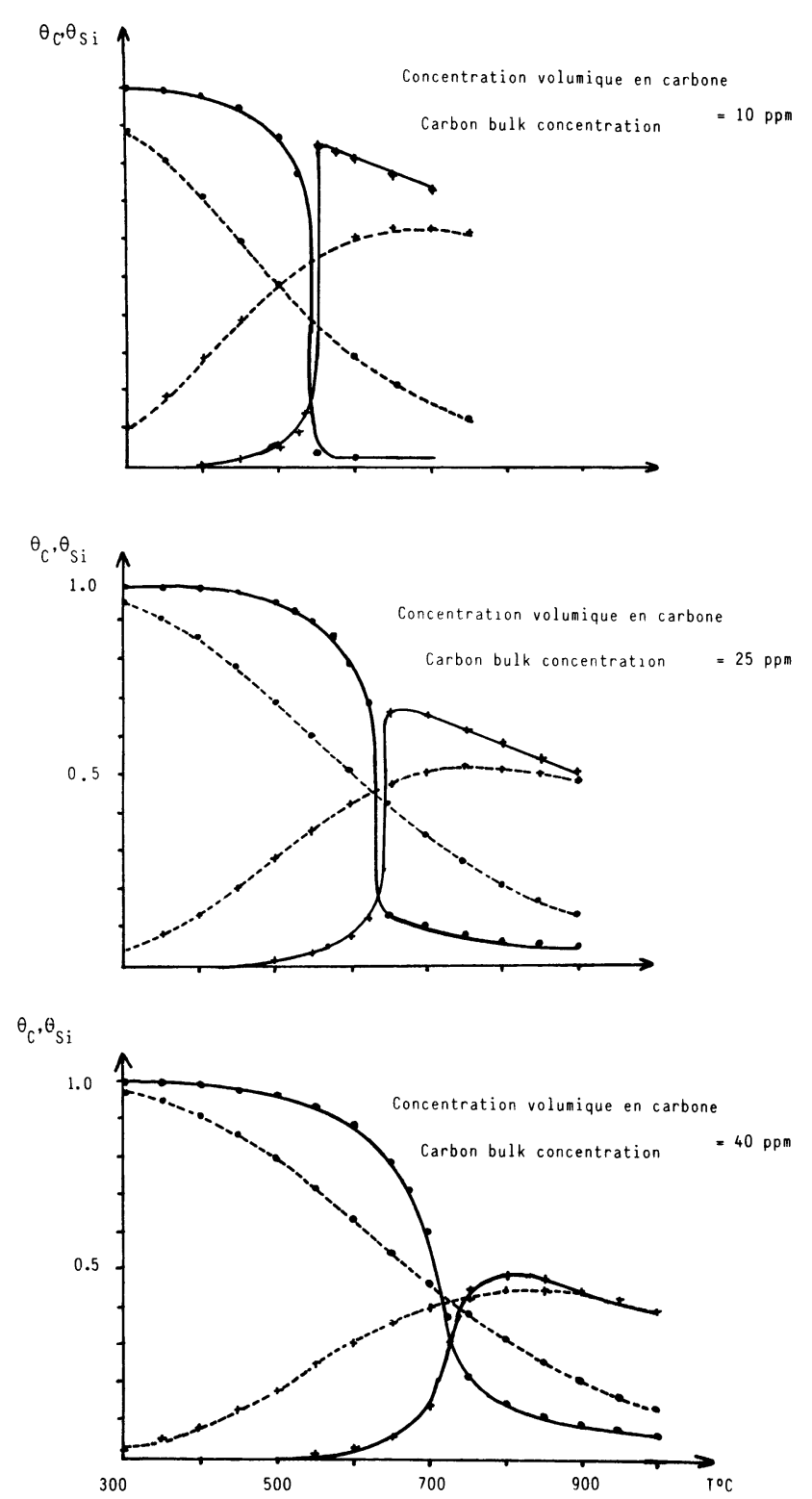

b)

[a) Experimental curves showing the variation of carbon $\theta_{\mathrm{C}}($.$) and silicon \theta_{\mathrm{Si}}(+)$ coverages with temperature for three different bulk concentrations after de Rugy [22]. b) Calculated curves according to Guttmann's model and giving the variation of carbon $\theta_{\mathrm{C}}($.$) and silicon \theta_{\mathrm{Si}}(+)$ coverages with temperature for carbon bulk concentration $=10,25$ and $40 \mathrm{ppm}$. Curves with dashed lines corresponds to $\alpha^{\prime}=0$.] 
tution; les courbes en pointillés étant relatives à $\alpha^{\prime}=0$ (absence d'interactions) pour les mêmes valeurs de $\Delta G_{\mathrm{C}}^{0}$ et $\Delta G_{\mathrm{Si}}^{0}$. On voit que ces derniers ne coïncident pas avec les courbes expérimentales, ce qui montre bien que les résultats ne peuvent être interprétés par un modèle de type Langmuir-McLean étendu aux solutions ternaires.

Par contre, nous constatons un bon accord entre les courbes calculées à l'aide du modèle de Guttmann et les courbes expérimentales. En particulier, le modèle rend bien compte du fait que la température de dissolution du carbone augmente avec la concentration volumique de celui-ci. En outre, le calcul permet d'estimer les concentrations en carbone $C_{2}$ et $C_{3}$ comme étant égales respectivement à $25 \mathrm{ppm}$ et à 10 ppm en poids.

Il faut cependant remarquer, d'après les courbes calculées, que la dissolution du carbone se produit sur un intervalle de température de plus en plus restreint lorsque la concentration de celui-ci diminue ( $25^{\circ} \mathrm{C}$ pour $10 \mathrm{ppm}$ de carbone), ce qui n'est pas le cas pour les résultats expérimentaux.

\section{Conclusion.}

En conclusion, la ségrégation du carbone et du silicium à la surface (100) d'un alliage monocristallin de $\mathrm{Fe}-6$ at $\% \mathrm{Si}$ a été décrite en termes de compétition sur les sites de surface avec répulsion entre le carbone et le silicium. Le modèle de Guttmann relatif aux solutions ternaires de substitution constitue une approche adéquate de ce système et les courbes théoriques calculées à l'aide de ce modèle sont en bon accord avec les courbes expérimentales obtenues par de Rugy à l'aide de la spectroscopie d'électrons Auger.

La valeur du coefficient d'interaction $\alpha^{\prime}$ entre le carbone et le silicium à la surface du fer, qui remplit le mieux cet accord, est égale à - $23000 \mathrm{~J} / \mathrm{mole}$. De plus, l'enthalpie libre de ségrégation du carbone $\Delta G_{\mathrm{C}}^{0}$ trouvée (92000 J/mole) est en bon accord avec celle déterminée par Grabke sur la face (100) d'un monocristal de fer pur.

Enfin, les concentrations $C_{2}$ et $C_{3}$ de l'échantillon appauvri en carbone déterminées, en utilisant le modèle de Guttmann, ont été estimées à $25 \mathrm{ppm}$ et à $10 \mathrm{ppm}$ en poids respectivement.

\section{Bibliographie}

[1] Madon, R. J. and Shaw, H., Cat. Rev. 15 (1977) 69.

[2] Surry, P., Corr. Sci. 16 (1976) 879.

[3] Oudar, J. and MarCus, P., Appl. Surf. Sci. 3 (1979) 48.

[4] Marcus, P., Oudar, J. and Olefjord, I., Mater. Sci. Eng. 42 (1980) 191.

[5] McMahon, C. J., Mater. Sci. Eng. 42 (1980) 215.

[6] Guttmann, M., Philos. Trans. R. Soc. Lond. A 295 (1980) 169.

[7] SeaH, M. P., Acta Metall. 25 (1977) 345.

[8] Seah, M. P., Surf. Sci. 80 (1979) 8.

[9] Hondros, E. D. and SeaH, M. P., Int. Met. Rev. 222 (1977) 226.

[10] McMahon, C. J., Jr. and MarChut, L., J. Vac. Sci. Techn. 15 (1978) 450.

[11] McLean, D., Grain bondaries in Metal (Oxford Univ. Press) 1957, p. 118.

[12] Williams, F. L. and Nason, D., Surf. Sci. 45 (1974) 377.

[13] Abraham, F. F., Tsai, N. H. and Pound, G. M., Surf. Sci. 83 (1979) 406.
[14] Wynblatt, P. and Ku, R. C., Surf. Sci. 65 (1977) 511.

[15] HofmanN, S. and ERLeWEIn, J., Surf. Sci. 77 (1978) 591.

[16] Tyson, W. R., Acta Metall. 26 (1978) 1471.

[17] Stinespring, C. D., Surf. Sci. 150 (1985) 209.

[18] Guttmann, M., Surf. Sci. 53 (1975) 213;

GuttmanN, M., Mater Sci. Eng. 42 (1980) 227 ;

McMahon, C. J., Jr. and MARChUt, L., J. Vac. Sc. Techn. 15 (1978) 450.

[19] Darken, L. S., Trans. Metall. Soc. AIME 239 (1967) 90.

[20] Dumoulin, P. H. and Guttmann, M., Mater Sci. Eng. 42 (1980) 249.

[21] Darken, L. S., Trans. AIME 180 (1949) 430.

[22] De Rugy, Thèse, Paris 1982.

[23] Grabke, H. J., Paulitschke, W., Tauber, G. and VIEFHAUS, H., Surf. Sci. 63 (1977) 377.

[24] Kubaschewski, O. and Evans, E. LL., Metallurgical Thermo-Chemistry (Pergamon Press, Oxford) 1958.

[25] Nyquist, O., Lange, K. W. and Chipman, J., Trans. Am. Inst. Min. Engr. 224 (1962) 714. 